\title{
Integrated Diagnostics Design
}

\author{
Andreas Dinklage \\ Max-Planck-Institut für Plasmaphysik, EURATOM Association, \\ Wendelsteinstraße 1, 17491 Greifswald, Germany \\ Phone: (+49) 3834882328 \\ Fax: (+49) 3834882005 \\ E-mail: dinklage@ipp.mpg.de \\ Rainer Fischer \\ Max-Planck-Institut für Plasmaphysik, EURATOM Association, \\ Boltzmannstraße 2, 85748 Garching, Germany \\ and Robert Wolf \\ Max-Planck-Institut für Plasmaphysik, EURATOM Association, \\ Wendelsteinstraße 1, 17491 Greifswald, Germany
}

Total Number of Pages: 22

Total Number of Figures: 5

Please reproduce the color figures as $\mathrm{b} / \mathrm{w}$ figures.

Manuscript Accepted: Jun. 24, 2012

by Fusion Science and Technology, MS \#12-482

Dated: Jul. 02, 2012 


\title{
Integrated Diagnostics Design
}

\author{
Andreas Dinklage, Rainer Fischer, and Robert Wolf
}

\begin{abstract}
A new concept for the design of diagnostics for fusion devices is introduced. The concept is based on the combination of sets of different diagnostics to an integrated meta-diagnostic. The approach applies methods from Bayesian Experimental Design and allows for quantitative assessments of differing metadiagnostics. A specific example is the combination of interferometry and Thomson scattering data to discuss the capabilities of the proposed method. The approach is also to discuss minimum sets of diagnostics required to determine physics quantities with a given accuracy. This is relevant, e.g. for the control of reactor relevant scenarios such as in DEMO.
\end{abstract}




\section{MOTIVATION}

What is the value of a measurement? How should experiments be planned to optimize its outcome? In statistics, these issues give rise for a field of its own known as experimental design. There are plenty of statistical applications depending on methods to optimize information gains, e.g. clinical trials. But also in experimental science, there is obvious evidence that changing the settings of measurements can improve the outcome of an inference process.

Without any attempt for a deeper discussion, nuclear fusion experiments can be said to be much less statistical investigations rather than physics goal oriented experiments. A fusion experiment is usually performed to assess the validity of physics prediction or - in exploratory phases - to investigate novelties. The validity assessments are made to provide a physics basis for extrapolation of present day devices to future power plants which have the clear requirement to produce energy economically. Therefore, fusion experiments must ultimately go along with an improvement of the physical basis of a - generally speaking - complex, self-organized system affected by the interplay non-linear effects.

Consequently, the planning of fusion experiments is highly complicated and the planning of measurements (or diagnostics) is complicated as well. There are a couple of mutually amplifying reasons: a validated physics basis in fusion science require typically a set of different diagnostics and physical information. To give an example, transport analyses may employ kinetic measurements for the temporal and spatial variation of the electron and ion temperatures and densities along with measurements of the radial electric field. Physics knowledge coming into play are coordinate transformation of spatial dependencies inevitably needed to be involved in the inference to determine physical quantities, e.g. figures of merit for the energy confinement such as the energy confinement time or heat fluxes.

These fusion specific complications require scientific approaches for the planning task known in other fields of science as data fusion; see e.g. [1]. Comparing fusion to other fields like robotics or geospatial data analysis, fusion needs to cope with involved multi-scale problems (space: gyro radius to device dimensions, time: inverse growth rate of instabilities to L/R-times (L: plasma inductance, R: plasma resistance) of current relaxation up to thermal equilibration times of the plasma wall interaction). Moreover, given the target of reactor operation, fusion science is to prove the validity of predictive models to extrapolate present day findings to reactor scale devices. And experimental fusion science needs to link uncertain information from uncertain data and must 
employ physical models to perform the linkage. Hence, from a formal point of view, the analysis process may be considered as the combination of heterogenous, noisy sources of uncertain information along with the validity assessments of physical models. To resolve this problem, the concept of Integrated Data Analysis (IDA) [2-4] has been introduced for the joint interpretation of fusion diagnostics. IDA has successfully proven its capabilities to link experimental data and physical information in different fusion devices [5-8].

For this paper, it is worthwhile to reflect the basic concepts of IDA employed for data analysis before we introduce the design concept used here. The ultimate goal of IDA is to improve the quality of the inference process both by the inclusion of physical knowledge and by data pooling on the level of measured data. This is different to conventional approaches: usually, physics quantities from different measurements are combined on a level of analyzed quantities. To give an example, the conventional approach is to combine, e.g., reflectometry, lithium beam and Thomson scattering measurements by a combination of analyzed density profiles of each individual diagnostics. Differently, IDA attempts to find a most probable joint density profile being compliant with all data and physics assumptions required to link the data.

The benefit of IDA lies in the systematic use of correlations and interdependencies. This becomes apparent in cases for which the result depends on other quantities, e.g. in Thomson scattering the signal depends both on the electron density and the electron temperature. If one imposes additional information on the electron temperature, e.g. by measurements of the electron cyclotron emission, also the joint measurement of the density is affected, i.e. the uncertainty of the density measurement is potentially reduced [2]. As a probabilistic method, IDA relies on Bayesian inference, for a recent overview on Bayesian analysis in physical sciences see [9]. IDA employs the Bayesian interpretation of probability theory, i.e. the inference process may use incomplete knowledge which is encoded in probability density functions; the extent of which reflect the uncertainty on a parameter and the dimensionality of a probability density function reflects the number of uncertain quantities. Since all information can be employed, IDA has an enormous flexibility: although making potentially largest benefit by combining the measurements on a raw data level, also intermediate steps of integration involving separately analyzed data may turn out to improve the overall outcome. In terms of IDA, these intermediate results are interpreted as additional physical information. 
Getting back to the initial question of planning measurements, this paper is to discuss the concept of a concise combination of measurements and physical information for the design of future diagnostics. The basic idea follows the line of IDA: rather than combining separate diagnostics units, an Integrated Diagnostics Design (IDD) is to combine all possible measurements. We call the overall set of diagnostics a meta-diagnostic. In this sense, IDD applies the philosophy of data pooling for the design of experimental set-ups. Again, to explicitly distinguish IDD from IDA, IDA is a method for the analysis of data whereas IDD is an approach for the assessment and optimization of settings.

What is it good for? First, IDD is a conceptual value in itself. It supplements conventional approaches of designing diagnostics (with partial optimization of settings) and delivers alternatives of designs which can be assessed with regard to additional constraints, e.g. costs or engineering feasibility. Second, for future devices, IDD may help to assess the contribution of different measurements to the inference of a physical quantity, e.g. to compare the value of different temperature measurements or to exploit synergies from applying experimental techniques according to their specific strengths. Following the previously discussed example of density measurements, a density meta-diagnostic could consist of a high temporal resolution reflectometry and a high spatial resolution lithium beam measurement at the plasma edge and Thomson scattering and interferometry channels in the plasma core to combine the data to a full density profile [5]. IDD should be capable to tell which diagnostics are most appropriate for which part of the full information and should also be feasible to give figures for decision making. Background for such questions are considerations to decide, e.g. whether an additional channel for interferometry gives more benefit than a Thomson scattering channel. Third, IDD could be part of the solution for a feasible reactor operation control.

Operating fusion diagnostics in reactors can be expected to differ considerably from present day operation of diagnostics for physics exploration. If we expect both restricted diagnostics availabilities due to harsh environments and much reduced accessibility, the set of diagnostics needs to be reduced to the minimum set required for safe operation. Beyond such a monitoring, present day operation scenarios of tokamaks make it very likely that diagnostics will be also required for the control of a burning plasma. On the other hand, since a fusion reactor is supposed to have a small 
number of operation points, diagnostics are expected to have a much smaller scope than in present day experiments. The concept of meta-diagnostics could open the possibility to consider combinations of diagnostics, e.g. neutron measurements and magnetic sensors or other robust quantities, for being sufficient to control a working point of a burning plasma.

To reflect the state of developments in the line of this paper, Bayesian diagnostics design (BDD) has been employed to determine optimum choices for lines of sight of interferometers [10]. The approach employs techniques of Bayesian experimental design $[11,12]$. BED is the optimization of settings of a diagnostic unit employing the Bayesian interpretation of probability theory. Methodologically equivalent, BDD optimizes design parameters of diagnostics. Beyond the determination of optimum settings, in the interferometer example BDD also indicated how much the quality of the design decreased when the optimum setting has been left. The formal approach will be recapitulated in more detail below. Tools allowing for the assessment of the expected utility of an experiment are rarely but available for the planning of experiments to optimize the expected information gain [13]. The usual case is, however, that planning relies on the experience and knowledge of the operating personnel solely. For future experiments, such tools may help to decide even on the implementation or the comprehensiveness of specific diagnostics. To outline roughly the capabilities of such a tool, a calculation of the expected utility of experiments is required. It is expected that this quantity helps to decide whether and to what extent a given diagnostic set-up, respectively experiment is required to attain the goal of a physical study.

In the following section, the basic methodology is revised. A specific example is introduced subsequently. Finally, the results and implications are discussed.

\section{BAYESIAN EXPERIMENTAL DESIGN}

The method employed for Integrated Diagnostics Design is Bayesian experimental design. Technically, BED is to maximize an expected utility $\Xi$ with respect to some design parameters $\vec{\eta}$. A necessary condition for an optimal design is therefore

$$
\partial_{\vec{\eta}} \Xi(\vec{\eta}, \vec{\sigma}, I)=0
$$

where the parameters describing the errors $\vec{\sigma}$ of the expected data $\vec{d}$ are assumed to be given. The context information $I$ summarizes all assumptions entering the optimization and are kept here 
explicitly to document the optimization to be context dependent.

Following Lindley [11], the expected utility is due to an information gain $\mathcal{J}$ from the envisaged measurement marginalized over the expected data:

$$
\Xi(\vec{\eta}, \vec{\sigma}, I)=\int d \vec{d} P(\vec{d} \mid \vec{\sigma}, \vec{\eta}, I) \mathcal{J}(\vec{d}, \vec{\eta}, \vec{\sigma}, I)
$$

Since $\mathcal{J}$ depends on $\vec{d}, \vec{\eta}, \vec{\sigma}$ and $I$, the weight function for the marginalization with respect to $\vec{d}$ is the evidence of the envisaged measurement.

Being compliant with additivity, Lindley proposed to use a specific entropy measure, i.e. the mean Kullback-Leibler divergence with regard to all quantities $\vec{q}$ to be measured:

$$
\mathcal{J}(\vec{d}, \vec{\eta}, \vec{\sigma}, I)=\int d \vec{q} \quad P(\vec{q} \mid \vec{d}, \vec{\sigma}, \vec{\eta}, I) \log _{2}\left(\frac{P(\vec{q} \mid \vec{d}, \vec{\sigma}, \vec{\eta}, I)}{P(\vec{q} \mid I)}\right)
$$

where the information gain refers to the knowledge about $\vec{q}$ before a measurement (encoded by the prior $P(\vec{q} \mid I))$ and after a measurement (encoded by the posterior $P(\vec{q} \mid \vec{d}, \vec{\sigma}, \vec{\eta}, I)$ ). As the posterior is unknown, Bayes theorem is employed

$$
P(\vec{q} \mid \vec{d}, \vec{\sigma}, \vec{\eta}, I)=\frac{P(\vec{d} \mid \vec{q}, \vec{\sigma}, \vec{\eta}, I)}{P(\vec{d} \mid \vec{\sigma}, \vec{\eta}, I)} P(\vec{q} \mid I)
$$

to rephrase the expected utility finally to

$$
\Xi(\vec{\eta}, \vec{\sigma}, I)=\iint d \vec{d} d \vec{q} \quad P(\vec{q} \mid I) P(\vec{d} \mid \vec{q}, \vec{\sigma}, \vec{\eta}, I) \log _{2}\left(\frac{P(\vec{d} \mid \vec{q}, \vec{\sigma}, \vec{\eta}, I)}{\int d \vec{q} P(\vec{q} \mid I) P(\vec{d} \mid \vec{q}, \vec{\sigma}, \vec{\eta}, I)}\right)
$$

where $\log _{2}$ is used to measure $\Xi$ in bit. The prior plays the role to define the range of interest for an envisaged measurement, say, a temperature range. The prior is also a suitable way to weight regions of interest. The likelihood represents a virtual diagnostic and reflects a model of the measurement as elaborated in the following. Eq. 5 also reflects a technical difficulty: the evaluation of the expression requires a consecutive evaluation of three high-dimensional quadratures. The introduction of new parameters and quantities, therefore, leads with increasing number of optimization parameters to increasingly laborious numerical efforts. At this point, however, we note that this formulation of the Expected Utility consists of the likelihood and the prior only and can be evaluated for design tasks. 
Coming to practical evaluations, the explicit form of the likelihood is of interest. Assuming a measurement for which data $\vec{d}$ and related errors $\vec{\sigma}$ are determined, the likelihood is according to the maximum entropy principle a normal distribution:

$$
P(\vec{d} \mid \vec{q}, \vec{\sigma}, \vec{\eta}, I)=\prod_{j}\left(2 \pi \sigma_{j}^{2}\right)^{-1 / 2} \exp \left\{\sum_{j}-\frac{1}{2}\left(\frac{d_{j}-D_{j}}{\sigma_{j}}\right)^{2}\right\}
$$

where the index $j$ is running over all components of the data vector $\vec{d}$. The data predicted for the measurement, $D_{j}$, express the model of the measurement and are determined by means of the forward function $f$ which depends on the quantities $\vec{q}$ and the design parameters $\vec{\eta}$ under all stated assumptions $I$ :

$$
D_{j}=f\left(q_{j}, \vec{\eta}, I\right)
$$

\section{A. Experimental Design of Single Diagnostics Units}

For the examples discussed in the remainder, it is assumed that the quantities of interest are the electron density $n_{e}$ and electron temperature $T_{e}$.

$$
\vec{q}=\left\{n_{e}, T_{e}\right\}
$$

Furthermore it is assumed that the range of interest is given by some expectations for a future fusion device and is chosen to be:

$$
\begin{aligned}
& n_{e} \epsilon\left[0 \ldots 2 \times 10^{20} \mathrm{~m}^{-3}\right] \\
& T_{e} \epsilon[0 \ldots 20 \mathrm{keV}]
\end{aligned}
$$

a. Interferometry (IF) To illustrate the capabilities of BDD, a line-integrated density measurement is considered first. This example has been discussed in detail by Dreier et al. [10] to optimize the position of a single chord interferometer for Wendelstein 7-X.

Here, the forward function and errors read as:

$$
\begin{aligned}
D_{I F} & =k_{I F} \times \int_{L} n_{e}(z) d \ell \\
\sigma_{I F}^{2} & =\sigma_{I F 0}^{2}+r D_{I F}
\end{aligned}
$$


The interferometry data $D_{I F}$ are due to a phase shift proportional to the line integral of the electron density $n_{e}$ along the laser chord $L$. The constant $k_{I F}$ summarizes dependencies on natural constants, the laser wavelength and calibration factors provided the wavelength of the incident beam is far from the plasma cut-off wavelength. The design parameter is the chord position $\vec{\eta}=z$. The error of the measurement $\sigma_{I F}^{2}$ is assumed to consist of a constant contribution $\sigma_{I F 0}^{2}$ and a contribution dependent on the signal level multiplied by a factor $r$.

It is a fairly expectable result of the optimization that the most informative chord is a central one in circular geometry. This reflects a possibly anticipated optimum signal to noise ratio and would not be found if the error is assumed to be a relative error of the data only.

For the aforementioned study for Wendelstein 7-X, different optimization targets have been phrased in numerical conditions. E.g., the most informative chord for the localization of a density gradient is determined both by an interplay of signal-to-noise ratio and the regions of gradient variations. To illustrate this, Fig. 1 shows in its upper panel a poloidal cross section of a W7-X equilibrium in the envisaged plane of the interferometry. The density distribution is shaded to express real space variations. The lower panel of the figure shows the expected utility parameterized by two angles measured on a circle around the plasma in Fig. 1(a), both are measured clock-wise from a zero on the top of the circle. With this parametrization, any chord can be described by these two angles. The variation of the expected utility shows that channels crossing the plasma at about the flux surface with largest variability of expected density gradients (i.e. close to the plasma edge) have the largest expected utility. Among these, the chord traveling longest in real space through the plasma is calculated to be the most informative. It is notably, that this laser chord is the one among the selected edge chords with the highest signal-to-noise ratio. The maximum expected utility is indicated by the circle in Fig. 1(b), a corresponding chord is also shown in Fig. 1(a) for the purpose of illustration.

In addition to the demonstration of reasonable outcome, it is noteworthy that the expected utility also gives figures to indicate how much the optimum conditions are attained. This allows one to consider the robustness of an optimum choice. A further prominent feature of this approach is that it allows for a comparison of different diagnostics. This has been discussed in Ref. [10] for a multi-chord interferometer to consider which information gain can be attributed to newly introduced chords.

b. Thomson Scattering (TS) As a second example, the choice of spectral channels for a setup for incoherent Thomson scattering also discussed in Ref. [14] will be presented here. Assuming 
a polychromator set-up, the band-width of spectral filters are considered to be varied. Fig. 2(a) shows the relativistic scattering function $S\left(\lambda, T_{e}\right)$ for a laser wavelength of $\lambda=1064 \mathrm{~nm}$. Fig. 2(b) shows a set of three spectral model filters and the cut-off wavelengths $\lambda_{1}$ and $\lambda_{2}$ are the assumed variables. For each spectral channel, the modeled data are given by

$$
D_{T S}^{(j)}=k_{T S}^{(j)} \times n_{e}(x) \int \tau^{(j)}(\lambda) S\left(\lambda, T_{e}\right) d \lambda
$$

where the transmission function $\tau$ of the $j$-th filter weights the wavelength contribution for a given electron temperature $T_{e} . k_{T S}^{(j)}$ is a calibration factor again. The errors for the measurement are again assumed to be

$$
\sigma_{T S}^{2}=\sigma_{T S 0}^{2}+r D_{T S}
$$

complying with experiences from existent set-ups.

Fig. 2(c) shows the data for a given density indicating the specific effect of spectral filters. The filter cut-off wavelengths are chosen as shown in Fig. 2(b). Then, for channel \# 1, the signal barely varies for lower temperatures but decreases monotonously for $T_{e}>1 \mathrm{keV}$. Channel \# 2 has maximum data at about $T_{e} \approx 2 \mathrm{keV}$ while channel \# 3 shows signals almost monotonously increasing with $T_{e}$ except for a saturation at highest temperatures.

As an example for the wavelength filter cut-off optimization, calculations for given error parameters have been performed $\left(\sigma_{T S 0}^{2}=0.6, r=1 \%\right)$. Fig. 3(a) shows $\Xi$ as a function of the two cut-off wavelengths for the parameter range $\left[n_{e}, T_{e}\right]=\left[0 \ldots 2 \times 10^{20} \mathrm{~m}^{-3}, 0 \ldots 20 \mathrm{keV}\right]$. The parameter range of interest is reduced for Fig. 3(b) to $\left[n_{e}, T_{e}\right]=\left[0 \ldots 2 \times 10^{19} \mathrm{~m}^{-3}, 0 \ldots 1 \mathrm{keV}\right]$.

This comparison indicates that the expected utility depends on the range of interest: absolute figures for $\Xi$ increase with increasing ranges of interest roughly scaling with the volume of the range of interest. This needs to be considered for comparative studies. The optimum choice is on a broad maximum indicating the robustness against variations of the choices.

Finally, the maxima for the two cases differ somewhat but as anticipated: the smaller the temperature the closer the spectral channels are to be chosen towards the laser wavelength due to the temperature dependence of the scattering function.

The two model examples discussed in the present and the previous sub-sections show the capabilities of BDD for a design of fusion diagnostics. Now the concept of an integrated design will be introduced. 


\section{THE CONCEPT OF INTEGRATED DIAGNOSTICS DESIGN}

The concept of IDD is straightforward and conceptually simple: rather than optimizing individual diagnostics unit, IDD considers data sets as a whole. At this point, we introduce the concept of a meta-diagnostic as exactly the combined set of data channels, regardless which diagnostics contribute. Integrated Diagnostics Design employs Eq. 5 for the meta-diagnostics as previously discussed for individual units.

The expected benefit from this approach is sought to exploit synergies from a combined consideration. This is, as previously discussed, in line with the concept of Integrated Data Analysis (IDA) which benefits from a full data pooling. Different examples of IDA showed a quantitative benefit from the data pooling by using the information content of correlations [3].

To discuss the difference, we make use of the specific choice for the information gain, i.e. its additivity. Following the aforementioned terminology, the information gain for $k$ different diagnostic units being considered independently is

$$
\Xi^{(\mathrm{conv})}(\vec{\eta}, \vec{\sigma}, I)=\sum_{k} \Xi_{k}\left(\vec{\eta}, \sigma_{k}, I\right)
$$

in other words, the data space is spanned by independent subspaces:

$$
\vec{d}=\underbrace{\left[\left\{d_{1}, d_{2}, \ldots, d_{i}\right\}\right.}_{\text {diagnostics } 1}, \cdots \underbrace{\left.\left\{d_{j}, \ldots, d_{n}\right\}\right]}_{\text {diagnostics } k}
$$

Differently, IDD considers the full data-space as a whole:

$$
\vec{d}=\underbrace{\left\{d_{1}, d_{2}, \ldots, d_{i}, d_{j}, \ldots d_{n}\right\}}_{\text {meta-diagnostic }}
$$

allowing for arbitrary ordering. This is trivial but reflects the possibility to reorder the measurements to the most relevant data sub-sets forming a most informative meta-diagnostic for a given, finite number of measurements. The main difference of the formulation in Eqs. 16 and 17 is the capability of the latter to make use of correlations between the diagnostics represented by their respective data subspaces. If different measurements are completely independent, the IDD approach is not expected to deliver different results compared to the conventional approach. But different measurements from separate fusion diagnostics are usually not physically independent: different 
diagnostics may be partly affected by the same physics quantity or are linked by geometric information, such as the mapping on flux surfaces. A case for which the consideration of correlations results in an improved outcome is discussed in the next subsection.

\section{A. The Interferometer/Thomson-Scattering Meta-Diagnostic}

In order to apply the IDD concept a simple example of a meta-diagnostic is addressed. Specifically, the combination of interferometer and Thomson scattering channels is optimized. In order to visualize the quantities entering the expected utility $\Xi$, Fig. 4 shows the relevant probability distributions. The case shown in this figure assumes errors as discussed in the previous sections. To evaluate the line density signal of interferometry, parabolic profiles have been assumed. All densities and temperatures in Fig. 4 refer to central values.

To visualize the course of calculations in more detail, Fig. 4 shows the range of interest (prior distribution, upper left figure in Fig. 4), forward modeled data $D$ (right hand plots in the first row in Fig. 4), the likelihood functions of the expected data $d$ (four exemplifying cases in plots of the second up to the fifth row of Fig. 4) and the respective evidences (sixth row plots in Fig. 4). For the forward modeled data, the gray scale indicates that the expected data always linearly increase with the central density. For the Thomson scattering channels (columns indicated by TS), also a temperature dependence occurs corresponding to the integral of the scattering function weighted with the transmission characteristics of the polychromator channels. According to its forward function, the interferometer signal (column indicated by IF), $d$ does not show any temperature dependence since the forward function does not either. The likelihood distributions exemplify the explicit distributions for different values of expected data $d$. Again, the interferometer likelihood distributions are invariant against variations of the temperature. This is different for the Thomson scattering data: the likelihood function for individual Thomson scattering channels indicates the combinations of $T_{e}$ and $n_{e}$ which resulted in given data as indicated in front of the rows of the likelihoods. The widths of the likelihood probability distribution reflects the expected errors of the measurement. Finally, the evidence represents these likelihood distributions being marginalized with respect to the quantities. These distributions are employed to execute the quadrature required to evaluate $\Xi$ according to Eq. 5 .

The capabilities to measure the value of a measurement in figures of $\Xi$ is shown in Fig. 5. The plot shows the mean information gain of the Thomson-scattering/interfermeter meta-diagnostic 
(full lines) in comparison to the Thomson scattering only (dotted lines) and the meta-diagnostic without the Thomson scattering channel \# 1 (dashed dotted lines). Furthermore, the parameters of the error estimates $r$ are systematically varied over four orders of magnitude. The off-set error $s$ is used as the abscissa of the plots.

As a first result, $\Xi$ reflects a general, possibly anticipated tendency: the smaller the error (both $r$ and $s$ ), the more informative the measurement becomes. There is an exception for very large errors $(r=10 \%)$. Formally, the quadrature suffers for these large errors from large portions of expected data $D$ at the boundary region of the marginalization domains which may formally occur but are considered to be irrelevant (e.g. $D<0$ ). We kept this example to indicate that there are errors which may be that large that measurement give confusing results and quadrature problems may arise in the case of large errors. An extension of the marginalization domain mitigates this problem at the expense of higher computational effort.

A second finding, even more relevant to the determination of the value of a measurement is that the results are given in absolute figures of the information gain $\Xi$. For a given meta-diagnostic, this property allows statements about the value of apparent quality improvements. To discuss this in more detail, we consider the $r=1 \%$ case in Fig. 5 (plots squares). It can be readily seen that there is a minimum level of $s$ around a few times $10^{-2}$ below which the information gain cannot be improved. In other words, for this case the result imply that, e.g. using a detector with lower $s$ (for example a detector with lower dark current) would not improve the measurement.

Moreover, we can draw comparisons between differing set-ups. To exemplify this capability, we consider the $r=0.01 \%$ case in Fig. 5 (lines without attached symbols). The maximum information gain for Thomson-scattering only (dotted line for $s \rightarrow 0$ almost coinciding with the dashed-dotted line) is as informative as a full meta-diagnostic with errors larger than about $s>2 \times 10^{-2}$. This example shows that the assessment of information gain allows one to compare measuring capabilities of different set-ups quantitatively including and error levels.

Beyond criteria of design decisions, such an assessment of the information gain as shown in Fig. 5 is also valuable for the assessment of robustness of a meta-diagnostic in case of failures of components of the meta-diagnostic. This is relevant particularly for measurements in harsh environments expected for fusion reactors for which failures may occur more likely than in present day devices and for which a much restricted accessibility hinders an unproblematic maintenance. For instance, for the measurement set-up discussed here, we consider the two 'degraded' cases in more detail. First, the virtually coinciding cases for 'Thomson scattering only' 
and 'Interferometry without Thomson scattering channel \#1' (dotted and dashed-dotted lines for $r=0.01 \%, 0.1 \%$, and $1 \%$ ) indicate that the missing information from the first Thomson scattering channel could be compensated by an inclusion of interferometry data (compared with a fully functional Thomson scattering set-up). In a forthcoming study, it should be examined whether the inversion to yield the quantities of interest finally leads to the same results for the two 'degraded' cases or, e.g., the inversion uncertainties for $n_{e}$ and $T_{e}$ are differently affected.

Following this discussion, Fig. 5 also gives rise to a couple of issues for the proposed approach. First, it is to be outlined that the role of the target quantity should be kept in mind for the interpretation of the information gain: here, the interferometry does not contain any information about the electron temperature but constrains the joint electron-temperature and -density measurement. $\Xi$ will alter if $T_{e}$ is considered as the measurement target rather than $\left\{T_{e}, n_{e}\right\}$. Second, the information gain $\Xi$ is not trivially transformed to errors in the measurement of a quantity of interest. Since $\Xi$ relies on an information entropy, the comparison of set-ups with regard to $\Xi$ is a monotonous measure for the quality of a measurement. It is monotonously related to the improvement of measuring errors, but the relation can be nonlinear.

With regard to these studies, it should not be hidden that a large number of assumptions enter (error levels, distribution of expected data, fixed model parameters etc.) to keep the approach numerically tractable. The reason is that additional parameters add in general an additional dimension leading to quickly emerging computational costs. The experience about the quadrature in the presented examples is that the integration procedures need to be capable to treat highly peaked and broad distributions at the same time. Low dimensional projections helped to identify critical regions for the integrations.

\section{SUMMARY}

A new concept for the design of diagnostics based on an integrated optimization of metadiagnostics has been proposed and first studies have been performed. The method relies on the maximization of the mean information gain which can be expected from measurements for quantities of interest. The resulting expected utility $\Xi$ provides means to assess the value of measurements under parameter variations, e.g. to investigate the impact of improved accuracies encoded by parameters describing the error of a measurement. Furthermore, $\Xi$ allows to compare different set-ups in figures of the mean expected information gain. This results in criteria, e.g., to 
select between different set-ups or to provide input to assess improvements of a diagnostic. The IDD approach is expected to yield a higher information gain compared to the consideration of individual set-ups. It is sought that this benefit results from the consideration of all data as a whole similar to IDA.

The approach may result in high computational costs when the number of free parameters grows. This argument, however, is to be balanced to the savings in the diagnostic efforts and should be related to the overall effort of building a fusion power plant. The specific interpretation of $\Xi$ with regard to the uncertainty of a measurement is monotonous but may become non-linear as previous studies of BDD have shown.

A next step is to validate the approach. This could be done in a well diagnosed fusion device for which successively diagnostics channels are disregarded for the control of an actuator [15]. Such a validation qualifies the proposed method to discuss the problem to define a minimum set of diagnostics to operate a fusion power plant safely. This could be an approach to define a set of DEMO diagnostics compliant both with control requirements and technical constraints.

\section{ACKNOWLEDGEMENTS}

The authors appreciate interesting discussions with Wolfgang Treutterer. The authors are indepted to Heiko Dreier for the provision of calculations displayed in Figure 1. This work has been initiated in the German DEMO Working Group led by Hartmut Zohm. The authors would like to thank the organizers of the $7^{\text {th }}$ Workshop of Fusion Data Processing, Analysis and Validation (March, 26 th $_{-2} 8^{\text {th }}, 2012$ in Frascati, Italy) - namely Afra Romano, Lori Gabellieri, Maria Polidori, Guilia Bartolomei, Antonio Tucillo and Danilo Pacella - for their extraordinary warm preparation of a very fruitful meeting.

This work has been performed within an EFDA task (WP11-DIA-04-01-01/IPP/PS) funded by EURATOM. 


\section{REFERENCES}

[1] D.L. HALL, et al., Proc. IEEE 85, 6 (1997).

[2] R. FISCHER, et al., Rev. Sci. Instrum. 75, 4237 (2004).

[3] R. FISCHER, et al., Plasma Phys. Contr. Fusion 45, 1095 (2003).

[4] A. DINKLAGE, et al., Fusion Sci. Technol. 46, 355 (2003).

[5] R. FISCHER, et al., Fusion Sci. Technol. 58, 675 (2010).

[6] B. VAN MILLIGEN, et al., Rev. Sci. Instrum. 82,073503 (2011).

[7] G. VERDOOLAEGE, et al., IEEE Trans. Plasma Sci. 38, 3168 (2010).

[8] M.J. HOLE, et al., Rev. Sci. Instrum. 81, 10E127 (2010).

[9] U. VON TOUSSAINT, Rev. Mod. Phys. 83, 943 (2011).

[10] H. DREIER, et al., Rev. Sci. Instrum. 79, $10 E 712$ (2008).

[11] D.V. LINDLEY, Ann. Math. Stat. 27, 986 (1956).

[12] K. CHALONER, et al., Stat. Sci. 10, 273 (1995).

[13] R. PREUSS, et al., Europhys. Lett. 81, 55001 (2008).

[14] R. FISCHER, AIP Conf. Ser. 735, 76 (2004).

[15] W. TREUTTERER, priv. comm. (2012) 


\section{Figure captions}

Fig. 1: (a) Real space density distribution in the $\phi=194^{\circ}$ plane of Wendelstein 7-X. The line is to indicate an optimum line of sight as indicated in the lower plot. (b) Expected utility for all possible lines of sight parameterized by the start and end angle on a plasma circumventing circle in (a). The optimization target is the detection of an edge gradient.

Fig. 2:(a) Scattering function (scattering angle $\pi / 4$ for different electron temperatures (line codes as for (c)). (b) Exemplary transmission curves for three spectral channels; the edge wavelengths $\lambda_{1}$ and $\lambda_{2}$ are varied in the optimization study. (c) channel-wise wavelength weighted transmission for different temperatures. $n_{e} \int_{\mathrm{ch}} S\left(\lambda, T_{e}\right) \tau(\lambda) d \lambda$ is proportional to the forward modeled data.

Fig. 3: Expected utility $\Xi$ for two positions of a parabolic temperature and density profile. (a) shows a channel in the very center $(x=0)$, (b) at $x=0.8$ of the minor radius. The white circle indicates the region of maximum excepted utility. The sections indicate the ranges for the numerical integration, the upper half of the diagram has not been calculated since it corresponds to a change of $\lambda_{1}$ and $\lambda_{2}$. The remaining white patches correspond to almost singular integration kernels due to very small information entropies.

Fig. 4: Quantities relevant for the calculation of the expected utility $\Xi$ of the Interferometer/ThomsonScattering meta-diagnostic. The first plot in the upper row shows the range of interest distribution for the density and the temperature parameter represented by a constant, normalized distribution of $n_{e}, T_{e}$ values (range of interest). The gray scale for the remaining plots in the first row indicate the values of the forward modeled data D. The second up to the fifth rows show the likelihood distributions for given expected data $d$ as indicated in front of each individual row. The sixth row column the evidence as a function of the expected data $d$.

Fig. 5: Comparison of Expected Utilities from different data pooling approaches at different error levels. 
(a) Density Profile

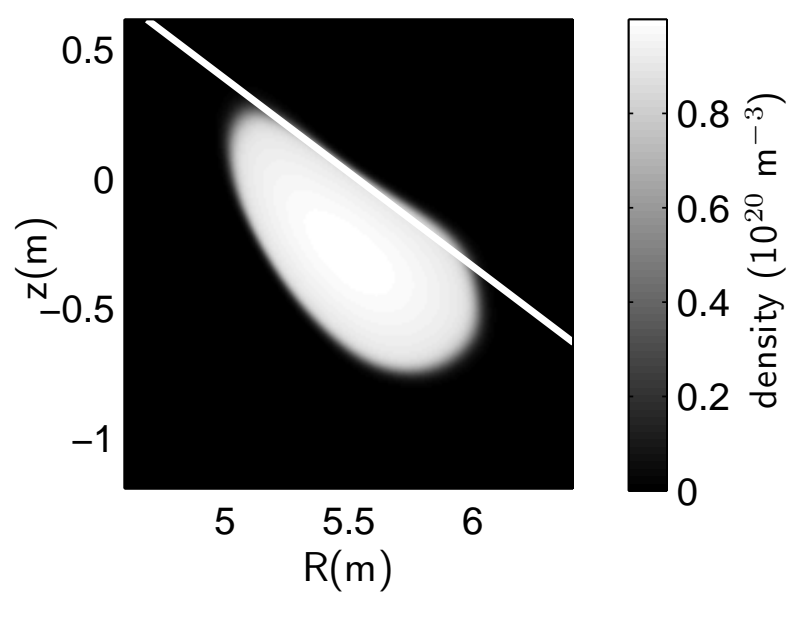

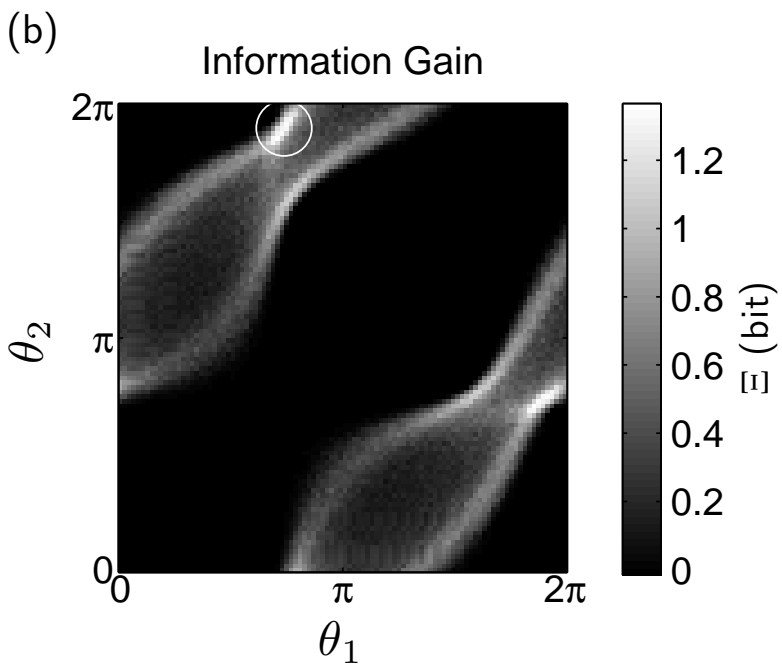

FIG. 1. 
(a)
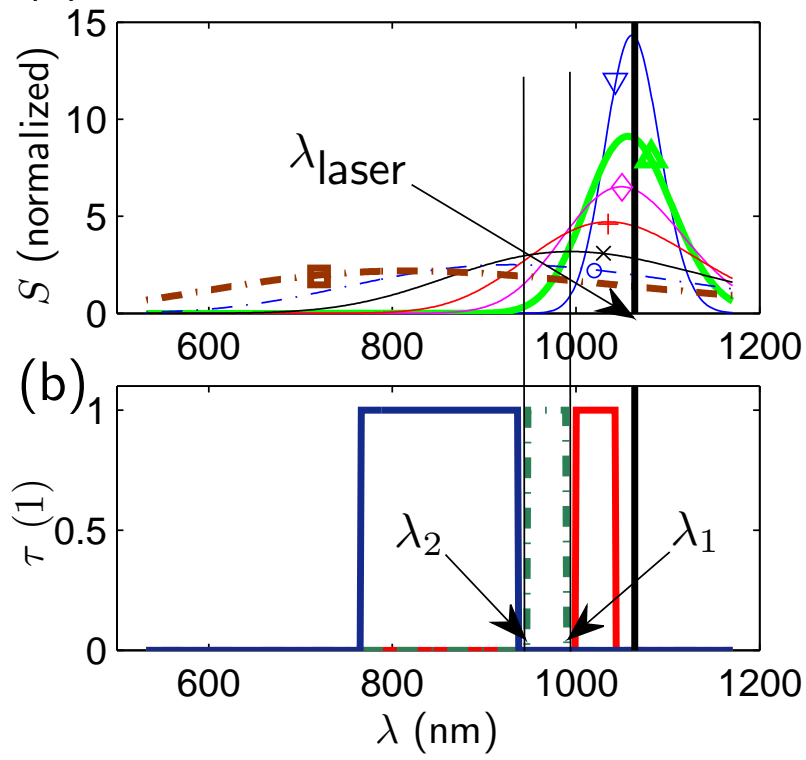

(c)

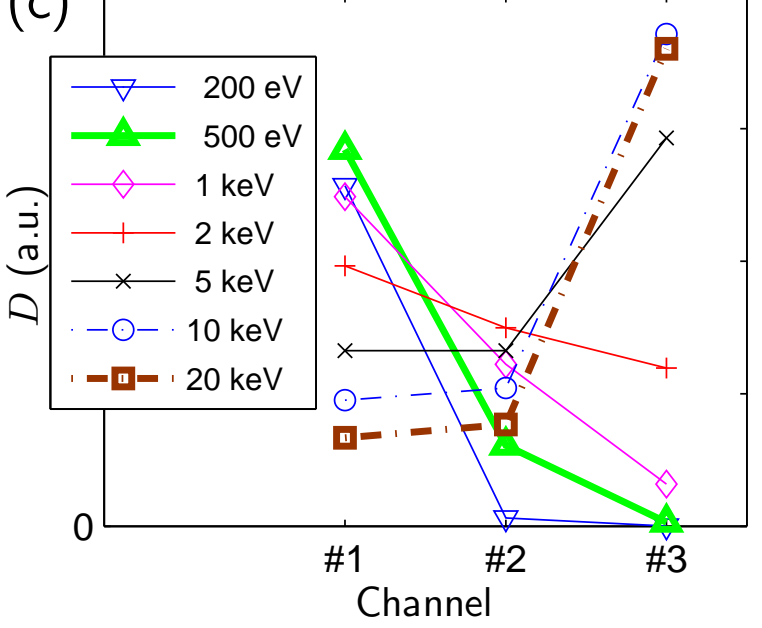

FIG. 2. 
(a)

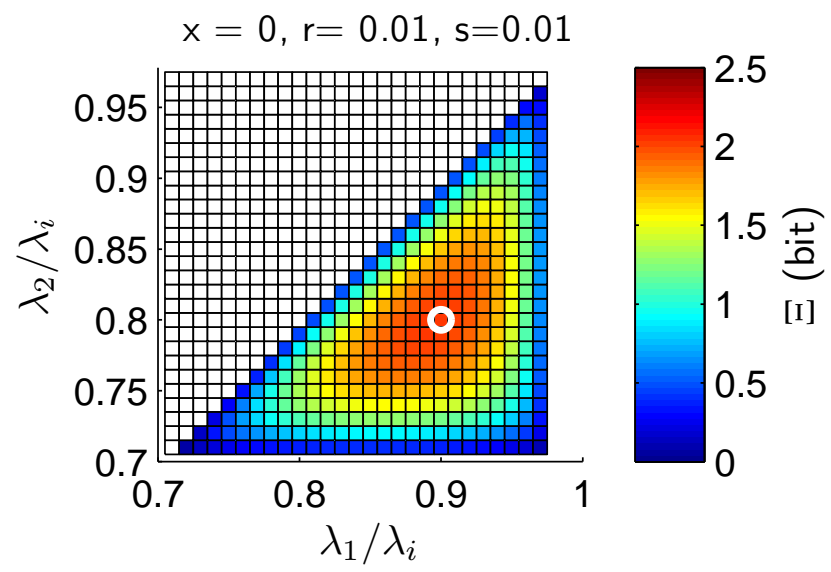

(b)

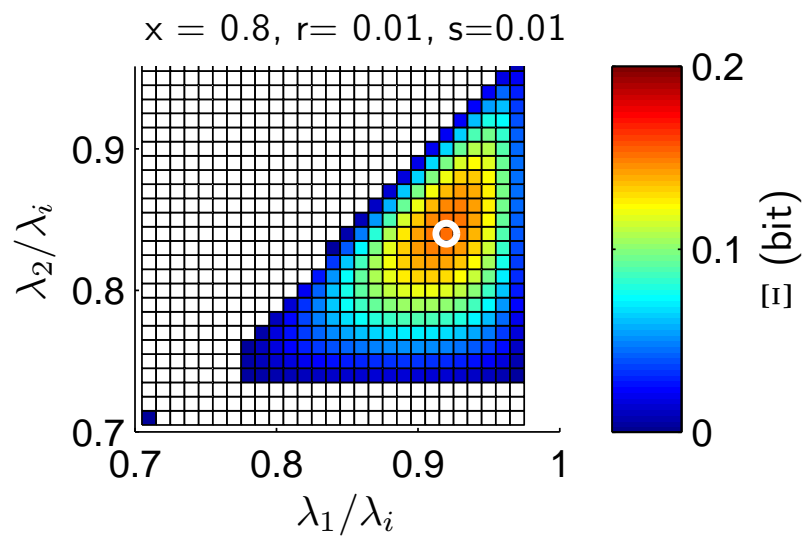

FIG. 3. 


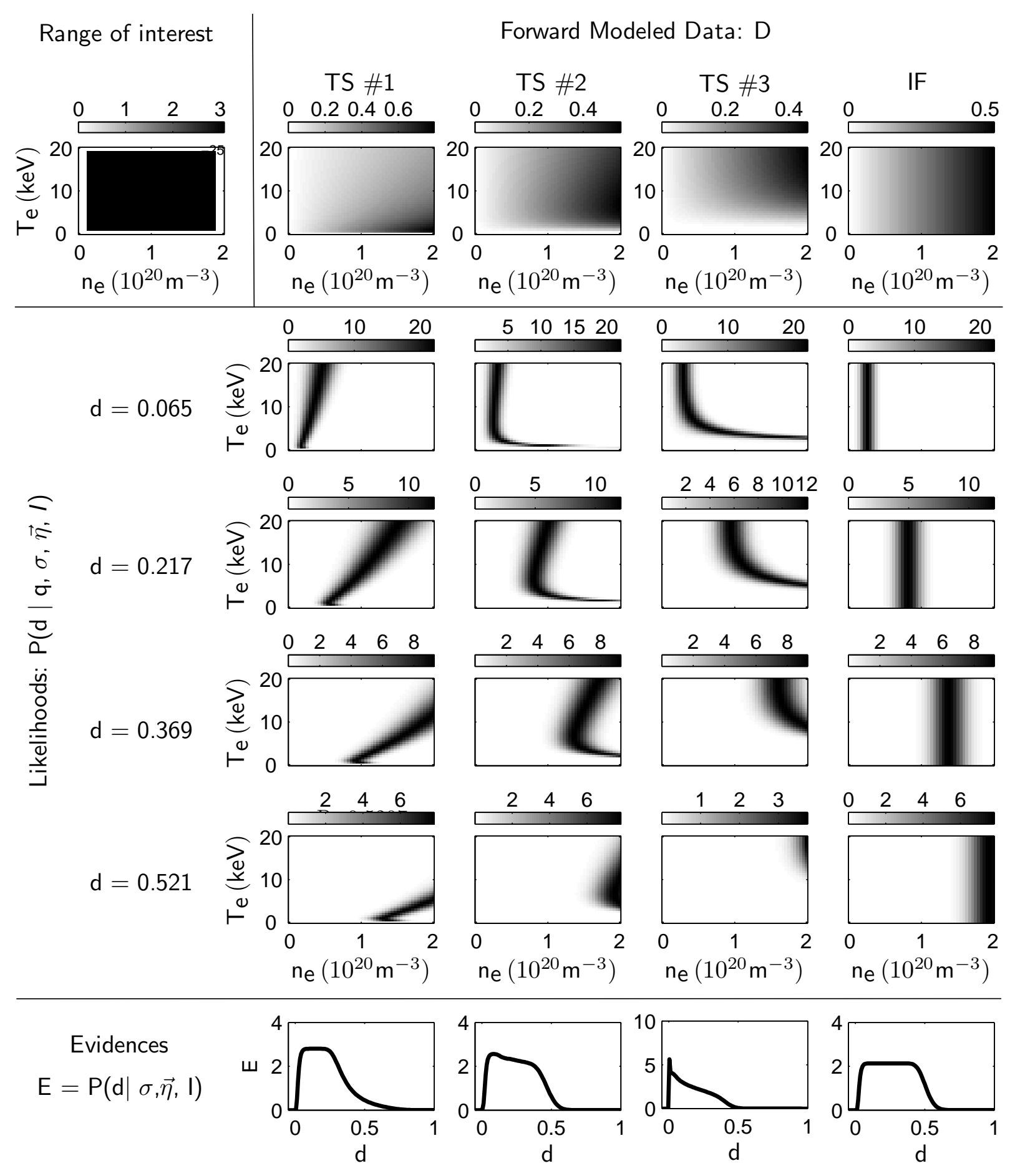

FIG. 4. 


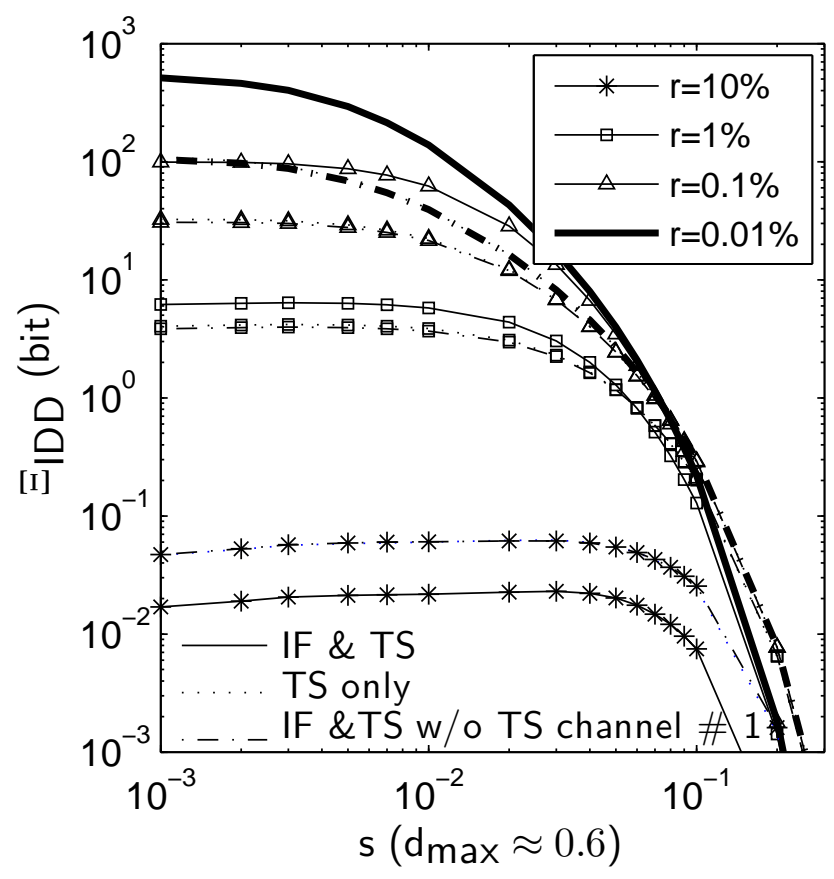

FIG. 5. 